\title{
Padrões alimentares e (in)segurança alimentar e nutricional no Programa Bolsa Família ${ }^{1}$
}

\author{
Food patterns and food and nutritional (in)security in the Bolsa Familia Program \\ Padrones alimentarios e (in)seguridad alimentaria y nutricional en el Programa \\ Bolsa Família
}

\author{
Daiane Roncato Cardozo \\ Sinara Laurini Rossato ${ }^{3}$ \\ Vera Mariza Henriques de Miranda Costa ${ }^{2}$ \\ Maria Rita Marques de Oliveira ${ }^{3}$ \\ Luiz Manoel de Moraes Camargo Almeida ${ }^{4}$ \\ Vera Lúcia Silveira Botta Ferrante ${ }^{2}$
}

Recebido em: 08/01/2019; revisado e aprovado em 31/10/2019; aceito em 29/11/2019

DOI: http://dx.doi.org/10.20435/inter.v21i2.2337

\begin{abstract}
Resumo: O Programa Bolsa Família (PBF) apresenta diversos efeitos na população com riscos e vulnerabilidades sociais, principalmente em relação à alimentação, educação e saúde. O objetivo foi identificar padrões alimentares de famílias beneficiárias ou não do PBF em município paulista, analisando sua associação com níveis de insegurança alimentar (IA). O estudo incluiu 150 famílias. Aspectos socioeconômicos, demográficos, antropométricos e de consumo alimentar foram avaliados por meio de questionários validados. A IA foi avaliada pela Escala Brasileira de Insegurança Alimentar (EBIA). Os padrões alimentares foram calculados utilizando a análise de Cluster. A associação entre a IA e o PBF com padrões alimentares foi testada utilizando o modelo de regressão de Poisson. Foram identificados três padrões alimentares: restrito, saudável e tradicional. O padrão restrito foi associado com menor escolaridade, histórico de anemia, IA moderada (IAM) ou IA grave (IAG) e maior idade média. Na análise não ajustada e ajustada para idade, as famílias com IAM ou IAG foram, respectivamente, 55\% e 57\% mais propensas a aderir um padrão restrito e $41 \%$ menos propensas a seguir um padrão saudável. Ter um padrão saudável ou tradicional foi relacionado à origem dessa população, revelando um elemento importante dentro do conceito de SAN: a soberania alimentar.
\end{abstract}

Palavras-chave: saúde pública; políticas públicas; renda familiar; hábitos alimentares; população carente.

Abstract: The Bolsa Familia Program (PBF) has several effects on the population with social risks and vulnerabilities, mainly in relation to food, education, and health. The objective of this study was to identify dietary patterns of beneficiary families or not of PBF in São Paulo municipality, analyzing their association with levels of food insecurity (FI). The study included 150 families. Socioeconomic, demographic, anthropometric, and food consumption aspects were evaluated through validated questionnaires. The FI was evaluated by the Brazilian Food Insecurity Scale (BFIS). Food patterns were calculated using Cluster analysis. The association between $\mathrm{FI}$ and PBF with dietary patterns was tested using the Poisson regression model. Three dietary patterns were identified: restricted, healthy and traditional. The restricted pattern was associated with lower educational level, anemia history, moderate FI (MFI) or severe FI (SFI), and higher mean age. In the unadjusted and adjusted for age analysis, the families with MFI or SFI were respectively 55\% and 57\% more likely to adhere to a restricted pattern and $41 \%$ less likely to follow a healthy pattern. Having a healthy or traditional pattern was related to the origin of this population, revealing an important element within the SAN concept: food sovereignty.

Keywords: public health; public policy; family income; eating habits; population in need.

Resumen: El Programa Bolsa Família (PBF) presenta diversos efectos en la población con riesgos y vulnerabilidades sociales, principalmente con relación a la alimentación, educación y salud. El objetivo fue identificar patrones alimentarios de familias beneficiarias o no del PBF en el municipio paulista, analizando

\footnotetext{
${ }^{1}$ Este trabalho contou com o apoio financeiro da Coordenação de Aperfeiçoamento de Pessoal de Nível Superior (CAPES), por meio da Bolsa de Doutorado da primeira autora.

${ }^{2}$ Universidade de Araraquara (UNIARA), Araraquara, São Paulo, Brasil.

${ }^{3}$ Universidade Estadual Paulista Júlio de Mesquita Filho (Unesp), Botucatu, São Paulo, Brasil.

${ }^{4}$ Universidade Federal de São Carlos (UFSCar), Buri, São Paulo, Brasil.
} 


\begin{abstract}
su asociación con niveles de inseguridad alimentaria (IA). El estudio incluyó a 150 familias. Los aspectos socioeconómicos, demográficos, antropométricos y de consumo alimentario fueron evaluados por medio de cuestionarios validados. La IA fue evaluada por la Escala Brasileña de Inseguridad Alimentaria (EBIA). Los patrones de alimentos se calcularon utilizando el análisis de clústeres. La asociación entre la IA y el PBF con patrones alimentarios fue probada utilizando el modelo de regresión de Poisson. Se identificaron tres patrones de alimentos: restringido, sano y tradicional. El patrón restringido fue asociado con menor escolaridad, historial de anemia, IA moderada (IAM) o IA grave (IAG) y mayor edad media. En el análisis no ajustado y ajustado para la edad, las familias con IAM o IAG fueron, respectivamente, el 55\% y el 57\% más propensas a adherirse a un patrón restringido y un $41 \%$ menos propensas a seguir un patrón sano. Tener un patrón sano o tradicional fue relacionado con el origen de esta población, revelando un elemento importante dentro del concepto de SAN: la soberanía alimentaria.
\end{abstract}

Palabras clave: salud pública; políticas públicas; renta familiar; hábitos alimenticios; población carente.

\title{
1 INTRODUÇÃO
}

As políticas de segurança alimentar e nutricional (SAN) no Brasil buscam ajustar-se à descentralização de políticas sociais com o objetivo de garantir o acesso aos direitos sociais básicos, como saúde, alimentação, educação, habitação, renda, entre outros aspectos, inseridos dentro do amplo conceito de SAN, o qual considera as dimensões de acesso, disponibilidade, estabilidade e utilização do alimento (BRASIL, 2006). Nesse contexto, programas de transferência de renda foram implementados para combater as desigualdades sociais, a fome e a pobreza no país, por meio da promoção da SAN, apresentando considerados efeitos na população (BRASIL, s.d.).

O Programa Bolsa Família (PBF), criado em 2004 pelo Governo Federal (GF), concede benefícios às famílias consideradas em situação de extrema pobreza, quando o rendimento por pessoa é inferior a $R \$ 85,00$, e de pobreza, quando superior a $R \$ 85,00$ e inferior a $R \$ 170,00$ (valores referentes a maio de 2016). O montante total pago às famílias depende do número de moradores menores de 18 anos de idade e de mulheres grávidas ou lactantes. Como resultado do programa, mais de 13 milhões de famílias deixaram a condição de pobreza², no entanto estudos mostram a prevalência de insegurança alimentar ainda em grande parte dessa população (INSTITUTO BRASILEIRO DE ANÁLISES SOCIAIS E ECONÔMICAS [IBASE], 2009; TRALDI; ALMEIDA; FERRANTE, 2012; CARDOZO, 2016; SPERANDIO; PRIORE, 2015; COTTA; MACHADO, 2013).

A pesquisa da Organização das Nações Unidas para Agricultura e Alimentação/Food and Agriculture Organization of the United Nations (ONU/FAO, 2014) sobre o estado de insegurança alimentar no mundo define a insegurança alimentar como a incapacidade de adquirir alimentos nutritivos e seguros de uma forma socialmente aceitável. Desse modo, para avaliar a insegurança alimentar de indivíduos, família ou uma população, alguns métodos têm sido utilizados em vários países, apresentando as discrepâncias entre eles (HU; WILLETT, 2002; ANDERSON, 1990; GALESI; QUESADA; OLIVEIRA, 2009; VANNIER-SANTOS, 2007; PÉREZ-ESCAMILLA; SEGALL-CÔRREA, 2008; LIGNANI et al., 2011). Por exemplo, nos países desenvolvidos como os Estados Unidos da América (EUA) e Portugal (VIEIRA et al., 2013), a avaliação da SAN está centrada na utilização e na estabilidade dos alimentos, enquanto nos países da América Latina e da África ela está concentrada nas vulnerabilidades sociais (JONES et al., 2013).

A Escala Brasileira de Insegurança Alimentar (EBIA) é o instrumento utilizado para avaliar a insegurança alimentar em ambientes populacionais, indagando ao responsável familiar questões subjetivas sobre o acesso e a disponibilidade do alimento, classificando as famílias em diferentes 
níveis: segurança alimentar (SA); e insegurança alimentar leve (IAL), moderada (IAM) e grave (IAG). A EBIA é amplamente aplicada no país devido a sua consistência interna e externa, mas reflete a perspectiva individual da insegurança alimentar (SEGALL-CÔRREA, 2007; SEGALL-CÔRREA; MARIN-LEÓN, 2009), ou seja, a percepção sobre ter a renda e conseguir ter acesso ao alimento em quantidade e qualidade, excluindo outros importantes indicadores, como histórico e hábitos alimentares, composição nutricional, entre outros, diante do atual processo de transformação dos padrões de alimentação da população mundial.

Dessa forma, este estudo teve como objetivo principal identificar padrões alimentares e analisar sua associação com níveis de (in)segurança alimentar e nutricional de famílias beneficiárias e cadastradas no Programa Bolsa Família no município de Araraquara, interior do estado de São Paulo (SP).

\section{METODOLOGIA}

Este estudo transversal abordou famílias cadastradas para o PBF no Centro de Referência de Assistência Social (CRAS) Parque São Paulo "Luiz Fernando Prudenciano", localizado no bairro Parque São Paulo, em Araraquar, SP, no período de 2015 a 2016. Uma sala foi reservada exclusivamente para a realização da coleta de dados com os participantes da pesquisa, mediante agendamento prévio por telefone para não haver conflito com o atendimento rotineiro da unidade.

O município apresenta aproximadamente 11.000 famílias cadastradas para os programas sociais do Governo Federal (Cadastro Único), sendo que, destas, 3.700 são beneficiárias do PBF. O CRAS Parque São Paulo compreende cerca de 700 famílias no Cadastro Único, sendo em torno de 400 famílias beneficiárias do Bolsa Família.

De acordo com o gestor local do PBF, o tempo mínimo para verificar o efeito do programa nas famílias era a partir de 2 anos de recebimento do benefício (experiência própria do gestor), além de que, no referido CRAS, as famílias eram compostas, em sua maioria, por até três membros. Desse modo, foram considerados como critérios amostrais, dentro da população beneficiária de 400 famílias, as famílias que recebiam o programa há dois anos ou mais, compostas por três membros, totalizando em 100 famílias. E, a fim de analisar possíveis diferenciações em ser ou não beneficiário do PBF, foi considerado como grupo controle, dentro da população de 700 famílias, as famílias não beneficiárias, mas que se encontravam cadastradas para participar do programa no Cadastro Único, também compostas por três membros, totalizando em 50 famílias.

Os padrões alimentares foram determinados com base na frequência da ingestão de 32 itens comumente consumidos pela população brasileira, identificados em outro estudo (IBASE, 2009). Foi utilizado o método de cluster K-means para classificar as famílias de acordo com a similaridade da frequência do consumo alimentar. Dois critérios foram utilizados para criar os padrões alimentares: a diferença estatisticamente significativa entre os três grupos de famílias na frequência semanal de consumo do alimento (valor- $p \leq 0,05$ no teste ANOVA) e a distribuição das famílias dentro de cada cluster de modo a alcançar um $n$ semelhante entre os três grupos. Como resultado, 11 dos 32 alimentos foram excluídos: arroz, feijão, amido de milho, farinha de milho, outras leguminosas, bebidas alcoólicas, soja, carne processada (presunto, bacon, salsicha, carne enlatada, salame), refrigerantes, chá e especiarias, pois a frequência de consumo (muito baixa) desses itens foi idêntica entre os grupos. 
Para fins de interpretação dos padrões, foram criadas três categorias: baixo consumo, quando o alimento foi consumido de zero a uma vez por semana; médio consumo, quando consumido de duas a quatro vezes por semana; e alto consumo, quando consumido cinco ou mais vezes por semana. Os padrões alimentares foram titulados com base nessas categorias e em outros estudos semelhantes sobre padrões alimentares (TAVARES et al., 2014; KANT, 2004; DESHMUKH-TASKAR et al., 2009; CUNHA et al., 2010; OLINTO et al., 2012; ASAKURA et al., 2015), sendo eles: padrão restrito, pois a frequência de consumo foi menor para a maioria dos alimentos; padrão saudável, pois, considerando que todas as famílias eram de baixa renda, a maioria dos itens alimentares era consumida em frequência moderada a alta, incluindo frutas, vegetais e leguminosas, com menor consumo de itens alimentares pouco saudáveis, como alimentos fritos, amiláceos e fast-foods; padrão tradicional, pois incluiu frequência similar de consumo de alimentos populares brasileiros, como leite e achocolatado, consumo moderado de frutas e legumes e menor consumo de doces, salgados e amiláceos.

O formulário semiestruturado, padronizado e validado por outras pesquisas, inclusive pelo Instituto Brasileiro de Análises Sociais e Econômicas (IBASE, 2009; TRALDI; ALMEIDA; FERRANTE, 2012; CARDOZO, 2016), contendo 64 questões sobre aspectos demográficos, socioeconômicos, de saúde, de consumo, hábitos alimentares e nível de insegurança alimentar e nutricional (EBIA), foi aplicado ao responsável da família pelo pesquisador. Cabe destacar que a escolha do alimento está relacionada à soberania alimentar, a hábitos e questões culturais e regionais, ou seja, à origem do indivíduo ou da família.

A EBIA incluiu 15 questões fechadas relacionadas à percepção de SAN, classificando as famílias em 4 níveis de (in)segurança alimentar: SA, IAL, IAM e IAG (SEGALL-CÔRREA; MARINLEÓN, 2009). As informações de peso e altura do responsável da família (Índice de Massa Corporal [IMC]) foram coletadas por meio de técnicas de medição padronizadas (WHO, 1995), por meio de uma balança digital da marca Tanita com capacidade para $150 \mathrm{~kg}$, em superfície lisa e nivelada, e de um estadiômetro (antropômetro vertical) portátil em MDF de 2 metros de altura, colocado em parede lisa e sem rodapé, com a régua em papel fornecida pela Rede de Defesa e Promoção da Alimentação Saudável, Adequada e Solidária (Rede SANS). As informações demográficas e socioeconômicas foram autorrelatadas e focadas nas características das famílias, como a região de origem, o número de crianças que vivem no domicílio, tempo de moradia; características do responsável, incluindo cor da pele, estado civil e nível de escolaridade; características econômicas, tais como a situação de trabalho, despesas mensais com alimentação, necessidade de doação de alimentos ou outros benefícios financeiros; condições de saúde e hábitos alimentares, como histórico de doença crônica, desnutrição, anemia, necessidade de assistência médica nos últimos seis meses e alimentos preferidos pelas crianças; entre outras.

As variáveis categóricas foram descritas em porcentagens e análise ordinal em médias e desvio padrão. As diferenças nas proporções de covariáveis de acordo com o padrão alimentar foram testadas utilizando o teste Qui-Quadrado de Pearson. As diferenças de idade média dos membros das famílias e do IMC do responsável da família foram testadas usando ANOVA. As análises de sensibilidade foram pré-formadas para avaliar potenciais fatores de confusão e ajustar-se ao modelo estatístico de análise. Quando a associação entre as variáveis explanatórias (indicadores demográficos, socioeconômicos, de hábitos e segurança alimentar) com a variável desfecho (padrões alimentares) ou exposição (PBF) não foi significativa, foram levados em consideração estudos que tenham encontrado associação entre essas variáveis (IBASE, 2009; 
GALESI; QUESADA; OLIVEIRA, 2009; PÉREZ-ESCAMILLA, 2008). Com base nisso, foram elaborados três modelos estatísticos para avaliar diferentes cenários.

A associação entre ser ou não beneficiário do PBF e a situação de SA/IAL ou IAM/IAG com padrão alimentar foram avaliadas pelo modelo de Regressão Generalizada, utilizando a razão de prevalência (PR) e o intervalo de confiança (IC) 95\%, e pelo modelo log-linear de Poisson, com estimador robusto, método híbrido de estimador de parâmetros e valores fixos para escala de parâmetros. Para a comparação entre as variáveis de exposição, foram utilizadas como categorias de referência o recebimento do PBF e os níveis de SA/IAL ou IAM/IAG. O desfecho foi analisado como uma variável dummy recodificando cada padrão alimentar em 1 e 0 . Todas as análises foram realizadas utilizando o SPSS 17.0 para Windows.

O trabalho obteve a aprovação do Comitê de Ética em Pesquisa (CEP) com Seres Humanos da Faculdade de Ciências Farmacêuticas, Universidade Estadual Paulista, Campus de Araraquara, São Paulo, Brasil, sob o parecer n. 847.071, de 14 de outubro de 2014.

\section{RESULTADOS E DISCUSSÃO}

A Figura 1 apresenta os padrões alimentares identificados de acordo com a frequência do consumo de cada alimento. O padrão alimentar restrito apresentou, em geral, a menor frequência de consumo para a maioria dos alimentos, com menor frequência no consumo de macarrão, farinha de mandioca, conservas, tubérculos e raízes e leguminosas; frequência média no consumo de biscoitos, bolos, ovos, frutas, sucos naturais, legumes, carne, pão, farinha refinada, lacticínios; e maior consumo de café. O padrão alimentar saudável incluiu a maior frequência de consumo de frutas e sucos naturais, legumes, carne, lacticínios, pão, farinha refinada e café; consumo médio de tubérculos e raízes, ovos, salgados, industrializados, milho, massas, farinha de mandioca; e menor consumo de fast-foods, frituras, pizza, peixe e frutos do mar, que são geralmente consumidos fritos no Brasil. O padrão alimentar tradicional incluiu maior frequência no consumo de leite e achocolatado; consumo médio de frutas, legumes, carne, café, pão e farinha refinada, biscoitos, bolos, conservas e ovos; e menor consumo de alimentos fritos, peixes e frutos do mar, pizza, hambúrguer, milho e farinha de mandioca. 
Figura 1 - Padrões alimentares entre beneficiários e não beneficiários do PBF (CRAS Parque São Paulo, Araraquara, SP, 2015/2016)

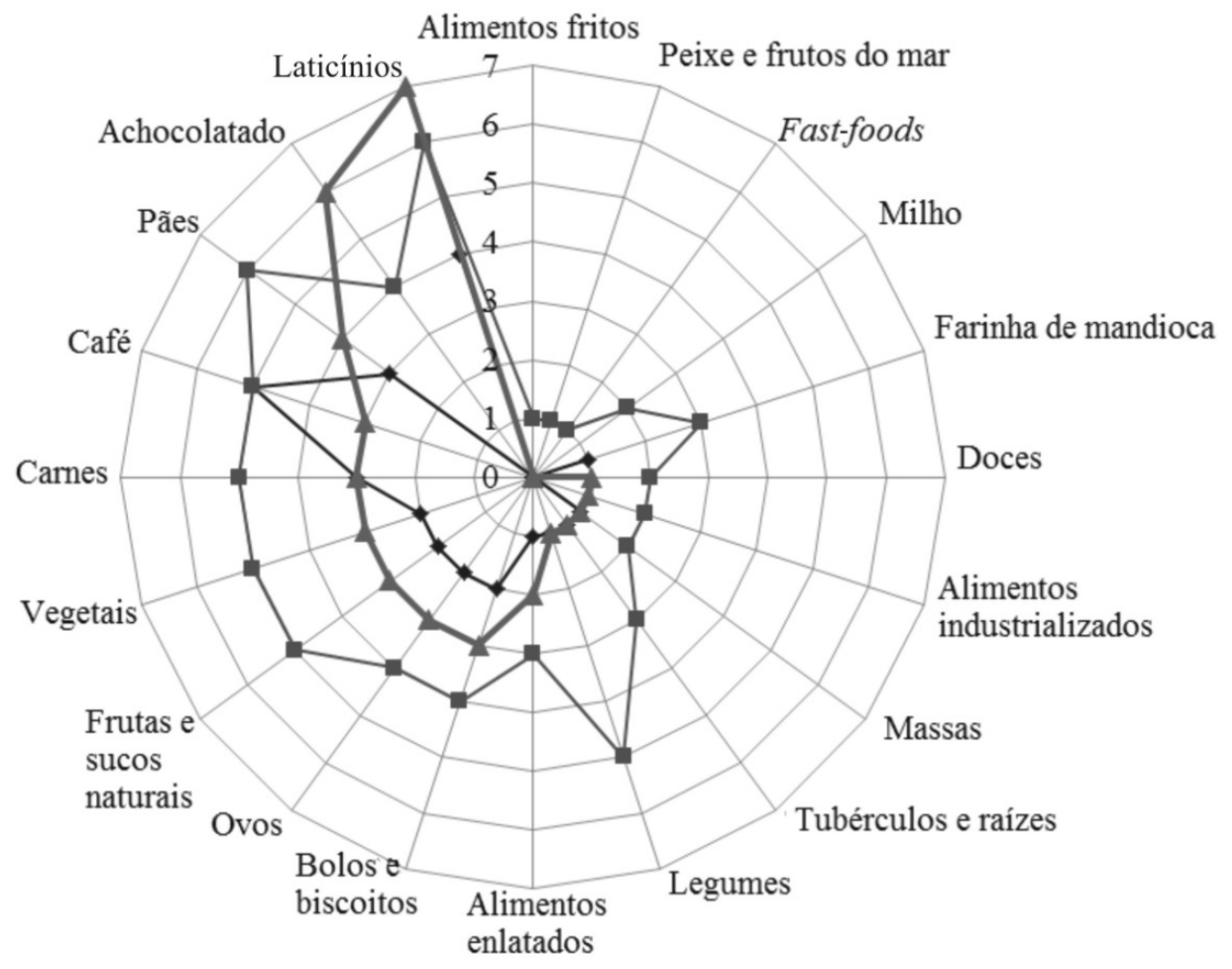

$\rightarrow$ Restrito

Saudável

\section{Tradicional}

Fonte: Cardozo (2016).

A Tabela 1 apresenta as características demográficas, socioeconômicas e antropométricas de acordo com o padrão alimentar. Na análise bruta, verificou-se que os participantes das regiões Norte, Nordeste e Centro-Oeste (regiões de origem) eram mais propensos a ter um padrão alimentar restrito (48\%) ou saudável (43\%) do que aqueles que eram do Sudeste ou do Sul, que aderiram a um padrão alimentar tradicional (44\%). Entre as famílias sem crianças e/ou jovens no domicílio, o padrão alimentar restrito foi mais frequente (81\%); já nas famílias com filhos (crianças e/ou adolescentes) o padrão alimentar tradicional foi mais frequente (38\%). Em relação à escolaridade dos titulares da família, 44\% que tinham menos de 10 anos de estudo apresentaram padrão alimentar restrito, ao passo que os titulares com maior escolaridade eram mais propensos ao padrão alimentar tradicional.

Quanto aos problemas de saúde relacionados à má alimentação, a anemia foi a única variável com associação estatisticamente significativa, sendo $49 \%$ das famílias que relataram anemia pregressa classificadas no padrão alimentar restrito. Além disso, houve diferença estatisticamente significativa entre os tipos de alimentos preferidos pelas crianças e jovens de acordo com o padrão alimentar, sendo que $45 \%$ das famílias com crianças que relataram preferir alimentos fritos foram classificadas no padrão alimentar tradicional, enquanto $45 \%$ das famílias com crianças que relataram preferir alimentos cozidos ou assados foram classificadas no padrão alimentar restrito. As demais variáveis não apresentaram associações significativas com os padrões alimentares. 
Tabela 1 - Características das famílias beneficiárias e não beneficiárias do PBF de acordo com os padrões alimentares (CRAS Parque São Paulo, Araraquara, SP, 2015/2016)

\begin{tabular}{|c|c|c|c|c|}
\hline & \multicolumn{3}{|c|}{ Padrões alimentares $(n=150)$} & \multirow[b]{2}{*}{ Valor-p } \\
\hline & $\begin{array}{l}\text { Restrito } \\
(n=59)\end{array}$ & $\begin{array}{l}\text { Saudável } \\
(n=40)\end{array}$ & $\begin{array}{c}\text { Tradicional } \\
\quad(n=51)\end{array}$ & \\
\hline Origem Norte/Nordeste/CO & $19(48)$ & $17(43)$ & $4(10)$ & $<0,01$ \\
\hline Origem Sudeste/Sul & $38(35)$ & $24(22)$ & $48(44)$ & \\
\hline Moradia própria (sim) & $28(38)$ & $22(30)$ & $24(32)$ & 0,70 \\
\hline Tempo de moradia $\leq 5$ anos & $27(35)$ & $23(30)$ & $27(35)$ & 0,70 \\
\hline Número de cômodos $\leq 4$ & $13(37)$ & $10(29)$ & $12(34)$ & 1,00 \\
\hline Cor da pele do titular branca & $37(39)$ & $26(27)$ & $33(34)$ & 1,00 \\
\hline Cor da pele preta/parda & $20(37)$ & $15(28)$ & $19(35)$ & \\
\hline Titular solteiro(a) & $25(42)$ & $17(29)$ & $17(29)$ & 0,50 \\
\hline Sem crianças ou jovens & $13(81)$ & $2(13)$ & $1(6)$ & $<0,01$ \\
\hline De 1 a 2 crianças ou jovens & $44(33)$ & $39(29)$ & $51(38)$ & \\
\hline Escolaridade $<10$ anos & $31(44)$ & $22(31)$ & $17(24)$ & 0,04 \\
\hline Auxílio em alimento (sim) & $19(37)$ & $9(18)$ & $23(45)$ & 0,08 \\
\hline Auxílio em dinheiro (sim) & $29(39)$ & $22(29)$ & $24(32)$ & 0,80 \\
\hline Titular empregado/autônomo & $29(33)$ & $26(30)$ & $33(38)$ & 0,30 \\
\hline Há problema crônico de saúde & $30(45)$ & $18(27)$ & $19(28)$ & 0,20 \\
\hline Desnutrição na família (sim) & $7(54)$ & $4(31)$ & $2(15)$ & 0,30 \\
\hline Obesidade na família (sim) & $16(52)$ & $8(26)$ & $7(23)$ & 0,20 \\
\hline Anemia na família (sim) & $24(49)$ & $7(14)$ & $18(37)$ & 0,03 \\
\hline Atendimento médico (sim) & $45(38)$ & $32(27)$ & $40(34)$ & 1,00 \\
\hline Famílias com SA/IAL & $35(33)$ & $34(32)$ & $37(35)$ & 0,07 \\
\hline Famílias com IAM/IAG & $22(50)$ & $7(16)$ & $15(34)$ & \\
\hline Gasto com alimentação < R\$ 250 & $20(50)$ & $7(18)$ & $13(33)$ & 0,08 \\
\hline Crianças preferem alimento frito & $12(24)$ & $16(31)$ & $23(45)$ & 0,03 \\
\hline Crianças preferem cozido/assado & $45(45)$ & $25(25)$ & $29(29)$ & \\
\hline
\end{tabular}

$\mathrm{CO}=$ Centro-Oeste; SA/IAL = segurança alimentar/insegurança alimentar leve; IAM/IAG = insegurança alimentar moderada/insegurança alimentar grave.

Fonte: Cardozo (2016).

A Tabela 2 apresenta as características das famílias de acordo com a inserção no PBF. Famílias que receberam o auxílio foram $84 \%$ mais propensas a viver em uma casa menor, $81 \%$ com maior probabilidade de o responsável pela família não ter companheiro(a), bem como foi 2,3 vezes maior a probabilidade de ter filhos morando na casa. Além disso, as famílias que receberam o benefício foram mais propensas a receber outro tipo de benefício financeiro (55\%) ou doação de alimentos (61\%) e ter o responsável da família empregado (trabalhando) do que aquelas famílias que não recebem o Bolsa Família. 
Tabela 2 - Características das famílias de acordo com o recebimento ou não do PBF (CRAS Parque São Paulo, Araraquara, SP, 2015/2016)

\begin{tabular}{|c|c|c|c|c|}
\hline & Não $(n=50)$ & $\operatorname{Sim}(n=100)$ & RP (IC 95\%) & Valor-p \\
\hline Origem Norte/Nordeste/Centro-Oeste & $15(38)$ & $25(62)$ & $1,20(0,73-1,91)$ & 0,50 \\
\hline Origem Sudeste/Sul & $35(32)$ & $75(68)$ & & \\
\hline Moradia própria & $24(30)$ & $50(70)$ & $0,90(0,57-0,41)$ & 0,60 \\
\hline Moradia alugada ou cedida & $26(40)$ & $46(60)$ & & \\
\hline Tempo de moradia $\leq 5$ anos & $29(40)$ & $48(60)$ & $1,39(0,83-2,10)$ & 0,30 \\
\hline Número de cômodos $\leq 4$ & $18(51)$ & $17(49)$ & $1,84(1,20-2,86)$ & 0,01 \\
\hline Titular solteiro(a) & $27(50)$ & $32(50)$ & $1,81(1,15-2,83)$ & 0,01 \\
\hline Titular com companheiro(a) & $23(30)$ & $68(70)$ & & \\
\hline Sem crianças/jovens & $11(69)$ & $5(31)$ & $2,36(1,55-3,60)$ & $<0,01$ \\
\hline De 1 a 2 crianças/jovens & $39(39)$ & $95(71)$ & & \\
\hline Escolaridade do titular $<10$ anos & $23(30)$ & $47(70)$ & $0,97(0,62-1,53)$ & 0,90 \\
\hline Titular empregado/autônomo & $26(30)$ & $62(70)$ & $0,76(0,49-1,20)$ & 0,20 \\
\hline Titular desempregado & $24(39)$ & $38(61)$ & & \\
\hline Desnutrição na família (sim) & $4(30)$ & $9(70)$ & $0,92(0,39-2,14)$ & 0,80 \\
\hline Obesidade na família (sim) & $8(30)$ & $23(70)$ & $0,73(0,38-1,34)$ & 0,30 \\
\hline Anemia na família (sim) & $12(20)$ & $37(80)$ & $0,65(0,38-1,31)$ & 0,10 \\
\hline Atendimento médico (sim) & $38(30)$ & $79(70)$ & $0,89(0,53-1,50)$ & 0,70 \\
\hline Auxílio em alimento (sim) & $11(20)$ & $40(80)$ & $0,55(0,31-0,98)$ & 0,03 \\
\hline Auxílio em dinheiro (sim) & $19(30)$ & $56(70)$ & $0,61(0,38-0,98)$ & 0,04 \\
\hline Gasto com alimentos $<\mathrm{R} \$ 250$ & $9(23)$ & $31(78)$ & $0,60(0,32-1,15)$ & 0,08 \\
\hline Famílias com SA/IAL & $31(29)$ & $75(71)$ & $0,68(0,43-1,06)$ & 0,10 \\
\hline Famílias com IAM/IAG & $19(43)$ & $25(57)$ & & \\
\hline Crianças preferem alimento frito & $0(0)$ & $51(100)$ & $2,02(1,66-2,47)$ & $<0,01$ \\
\hline Crianças preferem cozido/assado & $50(50)$ & $49(50)$ & & \\
\hline
\end{tabular}

$\mathrm{CO}=$ Centro-Oeste; SA/IAL = segurança alimentar/insegurança alimentar leve; IAM/IAG = insegurança alimentar moderada/insegurança alimentar grave; RP = razão de prevalência; IC = intervalo de confiança.

Fonte: Cardozo (2016).

Na Tabela 3, foram calculados o IMC do responsável da família e a idade média dos membros da família de acordo com os padrões alimentares e o recebimento do Bolsa Família. A idade média dos membros das famílias beneficiárias foi 4,7 anos menor que a das famílias não beneficiárias, e famílias que aderiram a um padrão alimentar restrito foram 7 anos mais jovens do que as famílias que foram classificadas como tendo um padrão alimentar tradicional. Não houve diferenças estatísticas de acordo com o IMC. 
Tabela 3- Diferença das médias de idade dos moradores e do IMC dos titulares de acordo com os padrões alimentares e recebimento ou não do PBF (CRAS Parque São Paulo, Araraquara, SP, 2015/2016)

\begin{tabular}{|c|c|c|c|c|c|c|}
\hline & \multicolumn{6}{|c|}{ Média \pm DP $(n=150)$} \\
\hline & \multirow[b]{2}{*}{$\begin{array}{c}\text { Amostra } \\
\text { total }\end{array}$} & \multicolumn{3}{|c|}{ Padrões alimentares } & \multicolumn{2}{|c|}{ PBF } \\
\hline & & $\begin{array}{l}\text { Restrito } \\
(n=59)\end{array}$ & $\begin{array}{c}\text { Saudável } \\
(n=40)\end{array}$ & $\begin{array}{l}\text { Tradicional } \\
\quad(n=51)\end{array}$ & $\begin{array}{c}\text { Sim } \\
(n=100)\end{array}$ & $\begin{array}{c}\text { Não } \\
(n=50)\end{array}$ \\
\hline $\begin{array}{l}\text { Idade média dos } \\
\text { moradores }\end{array}$ & $\begin{array}{c}27,7 \\
\pm 6,10\end{array}$ & $\begin{array}{c}26,3 \\
\pm 11,7\end{array}$ & $\begin{array}{c}24,8 \\
\pm 10,0\end{array}$ & $\begin{array}{c}19,3 \\
\pm 6,70\end{array}$ & $\begin{array}{c}21,9 \\
\pm 8,20\end{array}$ & $\begin{array}{c}26,6 \\
\pm 12,8\end{array}$ \\
\hline Valor-p & & & & $<0,01$ & & $<0,01$ \\
\hline IMC dos titulares & $\begin{array}{l}23,5 \\
\pm 10,2\end{array}$ & $\begin{array}{c}28,4 \\
\pm 6,50\end{array}$ & $\begin{array}{c}27,5 \\
\pm 5,40\end{array}$ & $\begin{array}{c}27,2 \\
\pm 6,10\end{array}$ & $\begin{array}{c}27,7 \\
\pm 6,10\end{array}$ & $\begin{array}{c}27,7 \\
\pm 6,10\end{array}$ \\
\hline Valor-p & & & & 0,60 & & 1,00 \\
\hline
\end{tabular}

$\mathrm{DP}$ = Desvio Padrão; PBF = Programa Bolsa Família; IMC/BMI = Índice de Massa Corporal $\left(\mathrm{kg} / \mathrm{m}^{2}\right)$.

Fonte: Cardozo (2016).

As famílias com insegurança alimentar relataram uma maior preocupação de acabar os alimentos no mês. Contudo, mesmo sendo minoria, alguns revelaram a falta de alimentos em determinado momento, mas não para as crianças.

A Tabela 4 mostra a associação do Bolsa Família e os níveis de segurança e insegurança alimentar com os padrões alimentares, contendo a análise não ajustada e quatro modelos diferentes de ajuste para potenciais fatores de confusão. Na análise não ajustada $(A)^{5}$, as famílias classificadas com IAM ou IAG apresentaram 55\% mais probabilidade de aderir a um padrão alimentar restrito e $41 \%$ menos provável de ter um padrão alimentar saudável em comparação com as famílias com IAL ou SA. Quando ajustado para idade média dos membros das famílias $(B)^{6}$, houve aumento da probabilidade de $57 \%$ das famílias com IAM ou IAG seguirem um padrão alimentar restrito e redução em $41 \%$ da probabilidade de ter um padrão alimentar saudável. No entanto, quando ajustado para os outros três modelos estatísticos $(C, D \text { e E })^{7}$, a associação desapareceu, o que indica o efeito positivo de variáveis de confusão ou que o tamanho da amostra era muito pequeno para permitir encontrar uma associação estatisticamente significativa.

\footnotetext{
${ }^{5}$ A) Modelo não ajustado.

${ }^{6}$ B) Modelo 1: Ajustado apenas para idade média dos moradores no domicílio.

${ }^{7}$ C) Modelo 2: Ajustado para renda mensal domiciliar, situação conjugal do titular, número de crianças na casa, auxílio sob forma de alimentos, auxílio sob a forma de dinheiro, situação de trabalho do titular, alimentos preferidos pelas crianças, IMC, idade média dos moradores do domicílio. D) Modelo 3: Ajustado para renda mensal domiciliar, situação conjugal do titular, número de crianças na casa, alimentos preferidos pelas crianças, IMC, idade média dos moradores do domicílio. E) Modelo 4: Ajustado para renda mensal domiciliar, número de crianças na casa, alimentos preferidos pelas crianças, IMC, idade média dos moradores do domicílio.
} 
Tabela 4 - Associações entre PBF, insegurança alimentar e padrões alimentares (CRAS Parque São Paulo, Araraquara, SP, 2015/2016)

\begin{tabular}{|c|c|c|c|c|c|c|c|c|}
\hline \multirow{2}{*}{ Padrões } & \multicolumn{2}{|c|}{ Restrito } & Valor-p & \multicolumn{2}{|c|}{ Saudável } & Valor-p & Tradicional & \multirow[t]{2}{*}{ Valor-p } \\
\hline & \multicolumn{2}{|c|}{$R P(I C$ 95\%) } & & \multicolumn{2}{|c|}{$R P$ (IC 95\%) } & \multicolumn{2}{|r|}{$R P$ (IC 95\%) } & \\
\hline PBF & Não & Sim & & Não & Sim & & Não Sim & \\
\hline \multirow[t]{2}{*}{ A) } & 1,00 & 1,48 & 0,08 & 1,00 & 1,03 & 0,90 & $1,00 \quad 0,64$ & 0,20 \\
\hline & \multicolumn{2}{|c|}{$(0,96-2,27)$} & & \multicolumn{2}{|c|}{$(0,65-1,62)$} & & $(0,40-1,00)$ & \\
\hline EBIA & \multicolumn{2}{|c|}{ SA/IAL IAM/IAG } & & \multicolumn{2}{|c|}{ SA/IAL IAM/IAG } & & SA/IAL IAM/IAG & \\
\hline \multirow[t]{2}{*}{ A) } & 1,00 & 1,55 & 0,05 & 1,00 & 0,59 & 0,04 & $1,00 \quad 0,98$ & 0,90 \\
\hline & \multicolumn{2}{|c|}{$(1,00-2,42)$} & & \multicolumn{2}{|c|}{$(0,35-0,97)$} & & $(0,62-1,54)$ & \\
\hline PBF & Não & Sim & & Não & $\operatorname{sim}$ & & Não $\quad$ Sim & \\
\hline \multirow[t]{2}{*}{ B) } & 1,00 & 1,25 & 0,03 & 1,00 & 1,02 & 0,90 & 1,00 & 0,10 \\
\hline & \multicolumn{2}{|c|}{$(0,79-1,97)$} & & \multicolumn{2}{|c|}{$(0,63-1,65)$} & & $(0,48-1,54)$ & \\
\hline EBIA & \multicolumn{2}{|c|}{ SA/IAL IAM/IAG } & & \multicolumn{2}{|c|}{ SA/IAL IAM/IAG } & & SA/IAL IAM/IAG & \\
\hline \multirow[t]{2}{*}{ B) } & 1,00 & 1,57 & 0,05 & 1,00 & 0,59 & 0,05 & $1,00 \quad 0,92$ & 0,90 \\
\hline & \multicolumn{2}{|c|}{$(0,99-2,49)$} & & \multicolumn{2}{|c|}{$(0,35-0,97)$} & & $(0,56-1,52)$ & \\
\hline PBF & Não & Sim & & Não & Sim & & ão $\quad$ Sim & \\
\hline C) & 1,00 & 0,91 & 0,70 & 1,00 & 1,18 & 0,60 & $1,00 \quad 0,85$ & 0,60 \\
\hline & $(0,52$ & $1,58)$ & & $(0,64$ & $2,19)$ & & $(0,47-1,54)$ & \\
\hline EBIA & SA/ & AL IAM/IAG & & & AL IAM/IAG & & SA/IAL IAM/IAG & \\
\hline C) & 1,00 & 1,46 & 0,10 & 1,00 & 0,70 & 0,20 & $1,00 \quad 0,93$ & 0,80 \\
\hline & $(0,89$ & $2,38)$ & & $(0,39$ & 1,21) & & $(0,56-1,54)$ & \\
\hline PBF & Não & Sim & & Não & Sim & & Não $\quad$ Sim & \\
\hline D) & 1,00 & 0,96 & 0,90 & 1,00 & 1,19 & 0,50 & $1,00 \quad 0,69$ & 0,20 \\
\hline & $(0,57$ & $1,64)$ & & $(0,65$ & $2,18)$ & & $(0,39-1,19)$ & \\
\hline EBIA & $\mathrm{SA} / \mathrm{IA}$ & IAM/IAG & & $\mathrm{SA} / \mathrm{IA}$ & IAM/IAG & & SA/IAL IAM/IAG & \\
\hline D) & 1,00 & 1,46 & 0,10 & 1,00 & 0,86 & 0,30 & $1,00 \quad 0,92$ & 0,80 \\
\hline & $(0,9$ & $2,38)$ & & $(0,4$ & $1,54)$ & & $(0,56-1,52)$ & \\
\hline PBF & Não & $\operatorname{Sim}$ & & Não & Sim & & Não $\quad$ Sim & \\
\hline E) & 1,00 & 0,90 & 0,70 & 1,00 & 1,20 & 0,50 & $1,00 \quad 0,86$ & \\
\hline & $(0,52$ & $1,54)$ & & $(0,66$ & $2,19)$ & & $(0,48-1,54)$ & 0,60 \\
\hline EBIA & $\mathrm{SA} / \mathrm{IA}$ & IAM/IAG & & $\mathrm{SA} / \mathrm{IA}$ & IAM/IAG & & SA/IAL IAM/IAG & \\
\hline E) & 1,00 & 1,46 & 0,10 & 1,00 & 0,68 & 0,20 & $1,00 \quad 0,92$ & 0,70 \\
\hline & $(0,90$ & $2,38)$ & & $(0,3$ & $1,18)$ & & $(0,56-1,52)$ & \\
\hline
\end{tabular}

$\mathrm{RP}=$ razão de prevalência $(1,00) ; \mathrm{IC}=$ intervalo de confiança.

Fonte: Cardozo (2016).

Com a identificação de três padrões alimentares consistentes neste estudo, pode-se demonstrar que famílias que não estavam recebendo o programa social Bolsa Família, classificadas com segurança ou insegurança alimentar, eram mais propensas a aderir um padrão alimentar restrito e menos propensas a seguir uma dieta saudável, independentemente da idade dos membros das famílias.

A população deste estudo apresentou características semelhantes à de populações de outros estudos no Brasil. Por exemplo, as famílias com titulares sem companheiro(a) que tinham maior número de crianças e jovens no domicílio e que recebiam outros benefícios em forma de dinheiro e doações de alimentos eram em sua maioria beneficiárias do programa (IBASE, 2009; 
PIMENTEL; SICHIERI; SALLES-COSTA, 2009; FERREIRA et al., 2014), o que mostra uma adequada focalização do programa. Embora, em outros estudos, as famílias beneficiárias tendam a estarem desempregadas, principalmente nas regiões Norte e Nordeste do país (IBASE, 2009), as famílias que receberam o Bolsa Família eram mais propensas a estarem trabalhando (informalmente) neste estudo.

A associação entre o nível de escolaridade mais baixo, o autorrelato de anemia pregressa, a preferência de alimentos cozidos e assados por crianças e jovens e a adesão a um padrão alimentar restrito é consistente com a tendência de populações de baixa renda no país. Em uma avaliação mais profunda da situação das famílias do Norte e Nordeste, consideradas regiões com taxas de pobreza mais elevadas ${ }^{3}$, essa associação consiste com o padrão alimentar restrito identificado, bem como com o padrão alimentar saudável encontrado nas famílias de origem do Sul e Sudeste do Brasil.

Deste modo, a adesão a um padrão alimentar tradicional ou saudável pode ser determinada pelas escolhas individuais, enquanto o padrão alimentar restrito, neste caso, é resultado das condições vulneráveis da família, principalmente relacionadas à renda, uma vez que a desnutrição não teve associação estatisticamente significativa. Isto revela um elemento importante dentro do conceito amplo de SAN, que diz respeito à cultura alimentar e autonomia para decidir que alimentos serão consumidos (soberania alimentar). Quando uma família ou indivíduo tem ingestão alimentar menor do que outros e isso está associado à origem das famílias, caracteriza-se uma privação do direito humano à alimentação. Além disso, a baixa ingestão de alimentos saudáveis, como frutas e verduras, e a alta ingestão de alimentos processados e de elevado teor calórico, como os fritos e industrializados, hambúrguer, salgados e fast-foods, têm sido associadas à renda das famílias (IBASE, 2009; HU; WILLETT, 2002; OLIVEIRA et al., 2011; POWEL; NGUYEN; HAN, 2012; PAN AMERICAN HEALTH ORGANIZATION [PAHO], 2015; FERREIRA; MAGALHÃES, 2017). Nesse sentido, o padrão alimentar restrito foi associado à ausência de crianças e jovens no domicílio e com maior média de idade dos membros das famílias a priorizar as escolhas alimentares na garantia de uma alimentação adequada.

Um estudo demonstrou que a aquisição de alimentos é fortemente influenciada por fatores geográficos (áreas urbanas e rurais), independentemente da renda (COELHO; AGUIAR; FERNANDES, 2009). Há indícios, por exemplo, de que o consumo de leite e derivados está relacionado com a segurança alimentar (SILVA et al., 2012; PANIGASSI et al., 2008). Neste estudo, a ingestão de leite foi menor nas famílias com padrão alimentar restrito. Assim, considerando tais resultados e evidências de outros estudos, a percepção de segurança alimentar e nutricional das famílias está fortemente associada às escolhas alimentares.

Por fim, assumem-se possíveis limitações desse levantamento, em relação a não considerar famílias domiciliadas na área rural, que possuem consumo e hábitos alimentares diferentes, marcadas pelas trajetórias geográficas, culturais e modos de vida (COELHO; AGUIAR; FERNANDES, 2009); e à obtenção de dados de dieta baseada em questionário de frequência alimentar qualitativo, que não viabilizou uma avaliação detalhada das quantidades ingeridas de cada alimento ou da ingestão de nutrientes, mas apenas da frequência de consumo. Além disso, não foi avaliada a questão do consumo dos jovens na merenda escolar.

Por outro lado, este estudo inova em identificar padrões de consumo alimentar de famílias atendidas e cadastradas para o maior programa social do país, o Bolsa Família, incluindo uma metodologia de análise exploratória, que abre brechas para pesquisas adicionais na área de 
políticas públicas voltadas à SAN da população em situação de risco e vulnerabilidades sociais e ao avanço nas questões de saúde pública, mostrando que estar em segurança ou insegurança alimentar não diz respeito apenas a ter renda e acesso a uma determinada quantidade e variedade de alimentos; poder de escolha, acesso aos serviços de saúde, educação alimentar e nutricional (práticas e hábitos) e outros valores implícitos também são determinantes para a SAN dessa população.

\section{CONSIDERAÇÕES FINAIS}

O padrão alimentar restrito foi relacionado com maior proporção de famílias classificadas com IAM ou IAG e a adesão a um padrão alimentar saudável ou tradicional esteve mais relacionada com a origem das famílias, demonstrando que a SAN está claramente relacionada com a escolha do alimento, principalmente em relação à soberania alimentar, hábitos e questões culturais e regionais que este implica.

Desse modo, populações inseridas em contexto de altas vulnerabilidades e riscos sociais, principalmente famílias que são foco de programas sociais, demandam uma rede de apoio e proteção social, de políticas e programas que abarquem todos os aspectos relacionados ao conceito amplo de SAN. Não basta olhar somente para a quantidade ou o tipo de alimento que o indivíduo ingere ou qualificar este em desnutrido ou obeso por medidas; as trajetórias, costumes, origem, preferências e, principalmente, condições de vida dessas famílias vão muito além de medidas antropométricas ou de ingestão da quantidade adequada de alimentos.

O Brasil tem políticas e programas consolidados na perspectiva integradora de ações entre educação, saúde, assistência e alimentação. No entanto são necessários também estudos que buscam avaliar a efetividade em âmbito local dessas ações, principalmente quanto à disseminação dos instrumentos disponíveis em âmbito nacional.

\section{REFERÊNCIAS}

ANDERSON, S. A. Core indicators of nutritional state for difficult-to-sample populations. The Journal of Nutrition, v. 120, sup. 11, p. 1559-600, nov. 1990.

ASAKURA, L.; SHIMADA, D. Y.; TOLEDO, A. D.; COELHO, L. C.; ANDREONI, S.; SACHS, A.; SILVA, C. V. D. Fatores relacionados aos padrões alimentares de indivíduos com síndrome metabólica que frequentam ambulatório de nutrição em São Paulo. Nutrire, São Paulo, v. 40, n. 3, p. 362-75, set. 2015.

BRASIL. Lei n. 11.346, de 15 de setembro de 2006. Cria o Sistema Nacional de Segurança Alimentar e Nutricional - SISAN com vistas em assegurar o direito humano à alimentação adequada e dá outras providências. Diário Oficial da União, Brasília, 2006.

BRASIL. Ministério do Desenvolvimento Social e Agrário. Programa Bolsa Família. [s.d.]. Disponível em: https://mds.gov.br/assuntos/bolsa-familia. Acesso em: 10 jul. 2017.

CARDOZO, D. R. Renda, consumo alimentar e estado nutricional como indicadores complementares à insegurança alimentar e nutricional no Programa Bolsa Família. 2016. 117 p. Tese (Doutorado em Alimentos e Nutrição)- Universidade Estadual Paulista, Araraquara, SP, 2016.

COELHO, A. B.; AGUIAR, D. R. D.; FERNANDES, E. A. Padrão de consumo alimentar no Brasil. Revista de Economia e Sociologia Rural, Brasília, v. 47, n. 2, p. 335-62, abr./jun. 2009. 
COTTA, R. M. M.; MACHADO, J. C. The Bolsa Família cash transfer program and food and nutrition security in Brazil: a critical review of the literature. Revista Panamericana de Salud Publica, v. 33, n. 1, p. 54-60, jan. 2013.

CUNHA, D. B.; ALMEIDA, R. M. V. R.; SICHIERI, R.; PEREIRA, R. A. Association of dietary patterns with BMI and waist circumference in a low-income neighbourhood in Brazil. British Journal of Nutrition, v. 104, n. 6, p. 908-13, set. 2010.

DESHMUKH-TASKAR, P. R.; O'NEIL, C. E.; NICKLAS, T. A.; YANG, S. J.; LIU, Y.; GUSTAT, J.; BERENSON, G. S. Dietary patterns associated with metabolic syndrome, sociodemographic and lifestyle factors in young adults: the Bogalusa Heart Study. Public Health Nutrition, v. 12, n. 12, p. 2493-503, dez. 2009.

FERREIRA, H. S.; SOUZA, M. E. C. A.; MOURA, F. A.; HORTA, B. L. Prevalência e fatores associados à Insegurança Alimentar e Nutricional em famílias dos municípios do norte de Alagoas, Brasil, 2010. Ciência \& Saúde Coletiva, Rio de Janeiro, v. 19, n. 5, p. 1533-42, 2014.

FERREIRA, V. A.; MAGALHÃES, R. Práticas alimentares de mulheres beneficiárias do Programa Bolsa Família na perspectiva da promoção da saúde. Saúde e Sociedade, São Paulo, v. 26, n. 4, p. 987-98, 2017.

FOOD AND AGRICULTURE ORGANIZATION OF THE UNITED NATIONS (FAO). The state of food insecurity in the world: strengthening the enabling environment for food security and nutrition. Rome: FAO, 2014.

GALESI, L. F.; QUESADA, K. R.; OLIVEIRA, M. R. M. Indicadores de segurança alimentar e nutricional. Revista Simbio-Logias, Botucatu, SP, v. 2, n. 1, p. 221-30, maio 2009.

HU, F. B.; WILLETT, W. C. Optimal diets for prevention of coronary heart disease. Jama, v. 288, n. 20, p. 2569-78, nov. 2002.

INSTITUTO BRASILEIRO DE ANÁLISES SOCIAIS E ECONÔMICAS (IBASE). 2a etapa da pesquisa Repercussões do programa Bolsa Família na segurança alimentar e nutricional das famílias beneficiadas. Rio de Janeiro: IBASE, 2009.

JONES, A. D.; NGURE, F. M.; PELTO, G.; YOUNG, S. L. What are we assessing when we measure food security? A Compendium and review of current metrics. Advances in Nutrition, Bethesda, Md, EUA, v. 4, n. 5, p. 481-505, set. 2013.

KANT, A. K. Dietary patterns and health outcomes. Journal of the Academy of Nutrition and Dietetics, v. 104, n. 4, p. 615-35, abr. 2004.

LIGNANI, J. B.; SICHIERI, R.; BURLANDY, L.; SALLES-COSTA, R. Changes in food consumption among the Programa Bolsa Família participant families in Brazil. Public Health Nutrition, v. 14, n. 5, p. 785-92, maio 2011.

OLINTO, M. T. A.; GIGANTE, D. P.; HORTA, B.; SILVEIRA, V.; OLIVEIRA, I.; WILLETT, W. Major dietary patterns and cardiovascular risk factors among young Brazilian adults. European Journal of Nutrition, v. 51, n. 3, p. 281-91, abr. 2012.

OLIVEIRA, F. C. C.; COTTA, R. M. M.; SANT'ANA, L. F. R.; PRIORE, S. E.; FRANCESCHINI, S. C. C. Programa Bolsa Família e estado nutricional infantil: desafios estratégicos. Ciência \& Saúde Coletiva, Rio de Janeiro, v. 16, n. 7, p. 3307-16, jul. 2011.

PAN AMERICAN HEALTH ORGANIZATION (PAHO). Ultra-processed food and drink products in Latin America: trends, impact on obesity, policy implications. Washington DC: PAHO, 2015. 
PANIGASSI, G.; SEGALL-CORRÊA, A. M.; MARIN-LEÓN, L.; PÉREZ-ESCAMILLA, R.; SAMPAIO, M. F. A.; MARANHA, L. K. Insegurança alimentar como indicador de iniquidade: análise de inquérito populacional. Cadernos de Saúde Pública, Rio de Janeiro, v. 24, n. 10, p. 2376-84, out. 2008.

PÉREZ-ESCAMILLA, R.; SEGALL-CORRÊA, A. M. Food insecurity measurement and indicators. Revista de Nutrição, Campinas, SP, v. 21, Suplemento, p. 15s-26s, jul./ago. 2008.

PIMENTEL, P. G.; SICHIERI, R.; SALLES-COSTA, R. Insegurança alimentar, condições socioeconômicas e indicadores antropométricos em crianças da Região Metropolitana do Rio de Janeiro/Brasil. Revista Brasileira de Estudos de População, v. 26, n. 2, p. 283-94, jul./dez. 2009.

POWEL L. M.; NGUYEN, B. T.; HAN, E. Energy intake from restaurants: demographics and socioeconomics, 2003-2008. American Journal of Preventive Medicine, v. 43, n. 5, p. 498-504, nov. 2012.

SEGALL-CORRÊA, A. M.; MARIN-LEÓN, L. Food security in Brazil: the proposal and application of the Brazilian Food Insecurity Scale from 2003 to 2009. Segurança Alimentar e Nutricional, Campinas, SP, v. 16, n. 2, p.1-19, 2009.

SEGALL-CORRÊA, A. M. Insegurança alimentar medida a partir da percepção das pessoas. Estudos Avançados, São Paulo, v. 21, n. 60, p. 143-54, maio/ago. 2007.

SILVA, C. C. S.; OLIVEIRA, K. B. B.; ALVES, A. S.; NEVES, J. A.; MODESTO, C. A. C.; VIANNA, R. P. T. Associação entre consumo alimentar e (in)segurança alimentar e nutricional em São José dos Ramos-PB. Brazilian Journal of Food Technology, Campinas, SP, v. 15, n. especial, p. 23-30, maio 2012.

SPERANDIO, N.; PRIORE, E. S. Prevalence of household food insecurity and associated factors among Bolsa Família program families with preschool in Viçosa. Epidemiologia e Serviços de Saúde, Brasília, v. 24, n. 4, p. 739-48, out./dez. 2015.

TAVARES, L. F.; CASTRO, I. R. R.; LEVY, R. B.; CARDOSO, L. O.; CLARO, R. M. Padrões alimentares de adolescentes brasileiros: resultados da Pesquisa Nacional de Saúde do Escolar (PeNSE). Cadernos de Saúde Pública, Rio de Janeiro, v. 30, n. 12, p. 1-13, dez. 2014.

TRALDI, D. R. C.; ALMEIDA, L. M. M. C.; FERRANTE, V. L. S. B. Repercussões do Programa Bolsa Família no município de Araraquara, SP: um olhar sobre a segurança alimentar e nutricional dos beneficiários. Interações, Campo Grande, MS, v. 13, n. 1, p. 23-37, jan./jun. 2012.

VANNIER-SANTOS, M. C. Indicadores de segurança alimentar e nutricional: uma análise das possibilidades e limites a partir da POF 2002-2003. 2007. 205 p. Dissertação (Mestrado em Estudos Populacionais e Pesquisas Sociais) - Fundação Instituto Brasileiro de Geogradia e Estatística, Escola Nacional de Ciências Estatísticas, Rio de Janeiro, 2007.

VIEIRA, V. L.; GREGÓRIO, M. J.; CERVATO-MANCUSO, A. M.; GRAÇA, A. P. S. R. Ações de alimentação e nutrição e sua interface com segurança alimentar e nutricional: uma comparação entre Brasil e Portugal. Saúde e Sociedade, São Paulo, v. 22, n. 2, p. 603-17, 2013.

WORLD HEALTH ORGANIZATION (WHO). Physical status: the use and interpretation of anthropometry. Geneva, Switzerland: WHO, 1995. 


\section{Sobre os autores:}

Daiane Roncato Cardozo: PhD em Desenvolvimento Territorial e Meio Ambiente pela Universidade de Araraquara (UNIARA). Doutora em Alimentos e Nutrição pela Universidade Estadual Paulista Júlio de Mesquita Filho (UNESP). Professora colaboradora do PPG em Desenvolvimento Territorial e Meio Ambiente da UNIARA. E-mail: daianecardozo@msn.com, Orcid: http://orcid.org/0000-0003-3172-0601

Sinara Laurini Rossato: Pós-doutora em Epidemiologia Nutricional na Universidade de Harvard. Doutora em Epidemiologia pela Universidade Federal do Rio Grande do Sul (UFRGS). Mestre em Saúde Coletiva pela Universidade do Vale do Rio dos Sinos (UNISINOS). Pesquisadora visitante do Departamento de Nutrição da Escola de Saúde Pública de Harvard. Professora do Programa de Pós-Graduação em Enfermagem da Universidade Estadual Paulista Júlio de Mesquita Filho (UNESP). E-mail: sinara.Irossato@gmail.com, Orcid: http://orcid.org/0000-0001-6966-927X

Vera Mariza Henriques de Miranda Costa: Livre-docente em Economia Brasileira e doutora em Economia pela Universidade Estadual Paulista Júlio de Mesquita Filho (UNESP). Docente do Programa de Mestrado Profissional em Engenharia de Produção da Universidade de Araraquara (UNIARA). E-mail: verammcosta@uol.com.br, Orcid: http://orcid.org/0000-0002-8217-0219

Maria Rita Marques de Oliveira: Doutora em Ciência dos Alimentos pela Universidade de São Paulo (USP). Mestre em Alimentos e Nutrição pela Universidade Estadual Paulista Júlio de Mesquita Filho (UNESP). Docente e pesquisadora do Departamento de Educação da Faculdade de Medicina da UNESP de Botucatu. E-mail: mrmolive.unesp@gmail.com, Orcid: http://orcid.org/0000-0003-1226-4364

Luiz Manoel de Moraes Camargo Almeida: Pós-doutor pela FEAGRI-UNICAMP na área de Políticas Públicas e Desenvolvimento Rural. Doutor em Sociologia pela Universidade Estadual Paulista Júlio de Mesquita Filho (UNESP). Mestre em Engenharia de Produção pela Universidade Federal de São Carlos (UFSCar). Docente e pesquisador da UFSCAr, Campus Lagoa do Sino, Buri. Diretor do Centro de Ciências da Natureza. E-mail: manoel77@yahoo.com.br, Orcid: http://orcid.org/0000-0003-3066-9170

Vera Lúcia Silveira Botta Ferrante: Pós-Doutora pelo Instituto de Investigações Sociais da Universidade Nacional do México (UNAM). Doutora em Sociologia pela Universidade Estadual Paulista Júlio de Mesquita Filho (UNESP). Coordenadora do Programa de Pós-Graduação em Desenvolvimento Territorial e Meio Ambiente. E-mail: vbotta@techs.com.br, Orcid: http://orcid.org/0000-0002-0679-3852 
